\title{
Diet-dependent Natriuretic Peptide Receptor $C$ expression in adipose tissue is mediated by PPAR $\gamma$ via long-range distal enhancers
}

\author{
Fubiao Shi ${ }^{1,5}$, Zoltan Simandi ${ }^{3,4,5 \S}$, Laszlo Nagy ${ }^{3,4,5}$, Sheila Collins ${ }^{1,2,5 *}$
}

${ }^{1}$ Division of Cardiovascular Medicine, Department of Medicine, Vanderbilt University Medical Center, Nashville, TN 37232, USA

${ }^{2}$ Department of Molecular Physiology and Biophysics, Vanderbilt University, Nashville TN 37232, USA

${ }^{3}$ Departments of Medicine and Biological Chemistry, Johns Hopkins University School of Medicine,

Johns Hopkins All Children's Hospital, Saint Petersburg, FL 33701, USA

${ }^{4}$ Institute for Fundamental Biomedical Research, Johns Hopkins All Children's Hospital, Saint Petersburg, FL 33701, USA

${ }^{5}$ Sanford Burnham Prebys Medical Discovery Institute, Orlando, FL32827 USA

$\S$ Present address: Department of Bioengineering, University of Pennsylvania, Philadelphia, PA 19104, USA.

*Corresponding author.

Running title: PPAR $\gamma$ controls Nprc expression in mouse adipocytes

Keywords: Nprc, PPAR $\gamma$, adipocyte, natriuretic peptide, gene expression, enhancer, obesity

\section{Correspondence Address:}

Sheila Collins, Ph.D.

Vanderbilt University Medical Center

342B Preston Research Building

2220 Pierce Avenue, Nashville, TN 37232

Tel: (615)-936-5863

Email: sheila.collins@vumc.org, sheila.collins@vanderbilt.edu 

available under aCC-BY-NC-ND 4.0 International license.

Nonstandard Abbreviations: NP: natriuretic peptides, cGMP: cyclic guanosine monophosphate, PKG:

protein kinase G, HFD: high-fat diet, ChIP-seq: Chromatin immunoprecipitation-sequencing, 3C:

chromosomal conformation capture assay. 


\begin{abstract}
In addition to their established role to maintain blood pressure and fluid volume, the cardiac natriuretic peptides (NPs) can stimulate adipocyte lipolysis and control the brown fat gene program of nonshivering thermogenesis. The NP "clearance" receptor C (NPRC) functions to clear NPs from the circulation via peptide internalization and degradation and thus is an important regulator of NP signaling and adipocyte metabolism. It is well appreciated that the Nprc gene is highly expressed in adipose tissue and is dynamically regulated with nutrition and environmental changes. However, the molecular basis for how Nprc gene expression is regulated is still poorly understood. Here we identified Peroxisome ProliferatorActivated Receptor gamma (PPAR $\gamma$ ) as a transcriptional regulator of Nprc expression in mouse adipocytes. During 3T3-L1 adipocyte differentiation, levels of Nprc expression increase in parallel with PPAR $\gamma$ induction. Rosiglitazone, a classic PPAR $\gamma$ agonist, increases, while siRNA knockdown of PPAR $\gamma$ reduces, Nprc expression in 3T3-L1 adipocytes. We demonstrate that PPAR $\gamma$ controls Nprc gene expression in adipocytes through its long-range distal enhancers. Furthermore, the induction of Nprc expression in adipose tissue during high-fat diet feeding is associated with increased PPAR $\gamma$ enhancer activity. Our findings define PPAR $\gamma$ as a mediator of adipocyte Nprc gene expression and establish a new connection between PPAR $\gamma$ and the control of adipocyte NP signaling in obesity.
\end{abstract}




\section{Introduction}

The cardiac natriuretic peptides (NP), including atrial NP (ANP) and B-type NP (BNP), were first discovered as factors in atrial extracts that evoked a strong decrease in blood pressure when injected into rodents (1). They are now known to be secreted from atrial cardiomyocytes in response to increases in blood volume and cardiac wall stress. However, in conditions of persistent high blood pressure, some reports suggest BNP can also be produced in the ventricle (2). Thus the most well established physiological function of NPs is to maintain blood pressure and fluid volume (3). More recently, NPs have also been shown to stimulate adipocyte lipolysis to liberate fatty acids from adipose tissue with a potency comparable to the catecholamines $(4,5)$. This places the heart in a position of being both a consumer of fatty acids as well as a regulator of their release from adipose tissue. Furthermore, work from our lab has demonstrated that NPs could also promote the thermogenic program of brown adipocytes by increasing the gene expression programs of mitochondrial biogenesis, fatty acid oxidation and the key thermogenic protein uncoupling protein-1 (UCP1) (6).

The physiological effect of the NPs is mediated by the cyclic guanosine monophosphate (cGMP) - protein kinase G (PKG) signaling cascade in target cells. There are two receptors for the cardiac NPs: NP receptor A (NPRA) and NP receptor C (NPRC). Binding of NPs to NPRA activates its intracellular guanylyl cyclase activity, increases cGMP production and triggers the PKG-dependent signaling cascade (7). In contrast, binding of NPs to NPRC, which lack an intracellular guanylyl cyclase domain, results in the internalization and degradation of the peptides (7). NPRC-mediated NP degradation is an important mechanism to modulate the available pool of NPs for target cell activation.

NPRC appears to be an important regulator of adipose tissue NP signaling and energy metabolism. In humans, increases in circulating NPs are associated with weight loss, while obese human subjects across ethnic groups with metabolic syndrome often show reduced circulating NPs and biological efficacy (e.g., elevated blood pressure) (8-11). This biological response to NPs depends on the relative amount of the guanylyl cyclase receptor NPRA to the 'clearance' receptor NPRC. In mice it was shown that genetic deletion of NPRC did not change the plasma levels of ANP and BNP, but their circulating half-life was substantially increased (12). In adipocytes, the levels of NPRC are dynamically regulated in response to nutritional and hormonal status. In obese humans and rodent models, the levels of NPRC are elevated in adipose tissue $(9,13-18)$. This is of physiological significance because it has been proposed that greater removal of NPs from circulation by NPRC in adipose tissue could explain what has been referred to as a 'natriuretic handicap' linking obesity and hypertension (9-11). We previously reported that deletion of Nprc specifically in adipose tissue increases NP signaling and protects against diet-induced obesity and insulin resistance (16). As a result, the level of NPRC in adipose tissues is crucial for NP action. In spite of this 
important biology, there is little known about how the Nprc gene is regulated. To tackle this problem, we used in vitro adipocyte culture and diet-induced obesity mouse models to investigate the mechanisms by which Nprc gene expression is regulated in adipocytes and adipose tissues. Here we describe our results which identified Peroxisome Proliferator-Activated Receptor gamma (PPAR $\gamma$ ) as a transcriptional regulator of Nprc expression and showed that the PPAR $\gamma$ enhancer activity in the Nprc gene is related to Nprc mRNA induction in response to high-fat diet feeding. Although there are probably additional factors to control Nprc gene expression, our data provide the first insights on Nprc transcriptional regulation and establish a new role for PPAR $\gamma$ to control adipocyte NP signaling and metabolism during obesity.

\section{Results}

\section{Regulation of Nprc expression in adipocytes by PPAR $\gamma$}

In efforts to understand how Nprc expression is regulated in adipocytes, we first examined the expression of Nprc over the time course of 3T3-L1 adipocyte differentiation. As shown in Fig. 1A, Nprc mRNA levels progressively increased throughout the differentiation process. Meanwhile, the expression of Ppary, the master regulator of adipocyte differentiation (19), and Fatty acid-binding protein 4 (Fabp4), a wellcharacterized PPAR $\gamma$ target gene (20,21), also increased over the course of differentiation (Fig. 1B, 1C). In line with this, although Nprc protein was not detectable in the preadipocytes, it increased along with the differentiation process, in parallel to the protein levels of PPAR $\gamma$ and its targets FABP4 and Adiponectin (ADIPOQ) (22) (Fig. 1D). When fully differentiated, adipocytes treated with rosiglitazone, a potent PPAR $\gamma$ agonist, for 6 hours showed a further increase in Nprc transcript levels, in a similar fashion to Fabp4 (Fig. 2A). In contrast, siRNA knockdown of Ppary resulted in a significant reduction of Nprc levels compared to the scramble siRNA control (Fig. 2B). Similarly, NPRC protein was also significantly reduced with PPAR $\gamma$ knockdown as expected, like the other two PPAR $\gamma$ targets FABP4 and ADIPOQ (Fig. 2C). These data suggested that PPAR $\gamma$ controls Nprc expression in adipocytes. We further confirmed this observation in NIH-3T3 fibroblasts that stably express PPAR $\gamma($ NIH-PPAR $\gamma)(23,24)$. In NIH-PPAR $\gamma$ cells, Nprc mRNA increased in response to rosiglitazone treatment as expected, though to a lesser extent than Fabp4. In contrast, in the parental NIH-3T3 cells that lack PPAR $\gamma$, Nprc as well as Fabp4 transcripts did not increase after rosiglitazone treatment (Fig. 2D). These data further support the conclusion that expression of Nprc is regulated by PPAR $\gamma$.

\section{Interaction of distal PPAR $\gamma$ enhancers with Nprc promoter in adipocytes}

To determine the mechanism by which PPAR $\gamma$ regulates Nprc expression, we next searched for PPAR $\gamma$ binding sites in the mouse Nprc locus by analyzing a previously published PPAR $\gamma$ ChIP-seq dataset from 
3T3-L1 adipocytes and mouse subcutaneous inguinal white adipose tissue (iWAT) (25). As shown in Fig. $3 \mathrm{~A}$, there were several PPAR $\gamma$ binding sites in the regions $-9 \mathrm{~kb},-44 \mathrm{~kb},-49 \mathrm{~kb},-54 \mathrm{~kb},-58 \mathrm{~kb},-62 \mathrm{~kb}$ and $71 \mathrm{~kb}$ upstream of the Nprc transcription start site. These PPAR $\gamma$ binding peaks were well harmonized between 3T3-L1 adipocytes and the iWAT depot. It was also noted that the PPAR $\gamma$ binding activity was higher in the iWAT of mice fed with high-fat diet, and further increased with rosiglitazone administration (Fig. 3A). These data suggest that the control of Nprc expression by PPAR $\gamma$ could be mediated by longrange enhancers.

To determine whether these PPAR $\gamma$ binding sites are directly involved in the regulation of Nprc expression, we utilized chromosomal conformation capture (3C) assays to examine the interaction of these enhancers with the proximal Nprc promoter (Fig. 3B, 4A). Among those HindIII-digested restriction fragments that contained PPAR $\gamma$ enhancers, only the $-9 \mathrm{~kb},-44 / 49 \mathrm{~kb}$ and $-54 \mathrm{~kb}$ fragments could form chimeric ligation products with the Nprc promoter fragment (Fig. 4B), suggesting that the distal enhancers in these three fragments could interact with the Nprc promoter by forming a looping structure, which is essential for the function of distal enhancer elements (26).

\section{Functionality of Nprc promoter and the distal PPAR $\gamma$ enhancers}

To determine the functionality of the Nprc promoter and these distal enhancers, we next cloned a $2.2 \mathrm{~kb}$ Nprc promoter fragment and the PPAR $\gamma$ enhancer fragments (as showed in Fig. 5A) into a luciferase reporter system to test their reporter activity in response to rosiglitazone treatment. The $N p r c-2.2 \mathrm{k}$ promoter did not show any luciferase activity in NIH-PPAR $\gamma$ cells in response to rosiglitazone (Fig. 5B). The $T K$ promoter and the Angptl4 enhancer were used as negative and positive controls, respectively, as described in a previous report (27). Among all the distal enhancer fragments tested, the $-49 \mathrm{~kb},-54 \mathrm{~kb}$ and $-62 \mathrm{~kb}$ PPAR $\gamma$ enhancer fragments showed significant increases in luciferase activities in response to rosiglitazone (Fig. 5C). Since the $-62 \mathrm{~kb}$ fragment did not interact with Nprc promoter (Fig. 4B), it may be that it participates in the regulation of a gene other than Nprc. Therefore, our further analysis focused on the $49 \mathrm{~kb}$ and $-54 \mathrm{~kb}$ fragments. More detailed sequence analysis showed that there were three putative PPAR $\gamma$ response element (PPRE) motifs within the $-49 \mathrm{~kb}$ fragment and one in the $-54 \mathrm{~kb}$ fragment (Fig. 5A). These four PPREs were further cloned into the luciferase reporter vector. Both the $-49 \mathrm{~kb}-\mathrm{P} 2$ and $-54 \mathrm{~kb}-\mathrm{P} 4$ elements showed increased luciferase activity with rosiglitazone treatment, illustrating that the $-49 \mathrm{~kb}-\mathrm{P} 2$ and $-54 \mathrm{~kb}-\mathrm{P} 4$ are functional PPAR $\gamma$ binding sites, although the other candidate sites in the $-49 \mathrm{~kb}$ region may function only in the context of the intact chromatin. Although the fold induction of the individual PPRE is modest, the agonist effect is very specific and reproducible, and these elements might work together within the context of intact chromatin in a cooperative or synergistic manner. Note that in the parental NIH-3T3 cells, which do no express PPAR $\gamma$, none of the enhancer and PPRE fragments, including 
the positive control Angptl4 enhancer, showed any luciferase activity after rosiglitazone. This is consistent with a specific role for PPAR $\gamma$ in NIH-PPAR $\gamma$ cells. Taken together, these data suggest that PPAR $\gamma$ controls Nprc gene expression through these long-range distal enhancers.

High-fat diet-induced Nprc gene expression in adipose tissue is associated with increased PPAR $\gamma$ enhancer activity

We and others have shown that Nprc expression is increased in rodent adipose tissue in response to highfat diet (HFD) feeding (16-18). Coupled with the observation that there is more PPAR $\gamma$ binding to these distal enhancers in the ChIP-Seq data (Fig 3A; ref 25), we next questioned whether the increase of Nprc expression in HFD-fed mice is mediated by PPAR $\gamma$. To test this hypothesis, we examined the $-49 \mathrm{~kb}$ and $54 \mathrm{~kb}$ PPAR $\gamma$ enhancer activities in adipose tissues by measuring their enhancer RNA (eRNA) synthesis via QPCR (28). Interestingly, the eRNA level of $-49 \mathrm{k}$ PPAR $\gamma$ enhancer was significantly increased in the brown adipose tissue (BAT) and iWAT of HFD-fed mice, in parallel to the changes in Nprc gene expression itself (Fig. 6A). The increase of -54k PPAR $\gamma$ eRNAs was also significant in iWAT but only marginally so in the BAT (Fig. 6A). The level of Fabp4 mRNA was slightly decreased in BAT, but its -5.4kb PPAR $\gamma$ eRNA was not altered with HFD feeding (Fig. 6B), suggested a tissue and locus-specific effect of PPAR $\gamma$ action. The level of Npra mRNA was modestly increased in BAT, and unchanged in iWAT with HFD (Fig. 6C). In sum, our results lead us to conclude that obesity increases Nprc expression in the adipose tissue through a PPAR $\gamma$-dependent mechanism, the effect of which is the suppression of NP signaling, decreased energy expenditure and impaired glucose homeostasis (Fig. 7).

\section{Discussion}

\section{Transcriptional regulation of $\mathrm{Nprc}$ in adipocytes by PPAR $\gamma$ via long-range distal enhancers}

Although the regulation of Nprc expression has been linked to glucose levels, insulin action, estrogen and adrenergic receptor signaling in previous reports (29-32), our current study is the first to identify PPAR $\gamma$, the master regulator of adipogenesis (19), as a transcription factor that directly controls Nprc expression in adipocytes. We showed that PPAR $\gamma$ stimulates Nprc expression though the action of its long-range distal enhancers rather than by direct binding at the proximal core promoter. We further demonstrated that induction of Nprc expression in adipose tissues by high-fat diet is also potentially mediated by PPAR $\gamma$. The PPAR $\gamma$ enhancer RNA synthesis, an indicator of enhancer activity that could be evaluated by QPCR (28), was significantly increased in parallel to Nprc mRNA expression.

In our study, we compared the expression of Nprc with another well-characterized PPAR $\gamma$ target Fabp4 $(20,21)$. The effect of PPAR $\gamma$ and its agonist rosiglitazone on Nprc and Fabp4 expression were not uniform 
in some instances. For example, under basal conditions without PPAR $\gamma$ agonist stimulation, the expression of Fabp4 was significantly higher in NIH-PPAR $\gamma$ cells than in the parental NIH-3T3 cells, but basal Nprc mRNA levels were comparable in these two cell types (Fig. 2D). This suggested that Nprc expression is more dependent on the action of a PPAR $\gamma$ ligand for maximal PPAR $\gamma$ activation $(33,34)$. In addition, PPAR $\gamma$ can bind to both the distal enhancer and the proximal promoter of Fabp4 $(21,35)$, which might be more potent than binding the distal enhancer alone; thus it is possible that Fabp4 expression could still be stimulated by PPAR $\gamma$ without its full activation by the ligand. In line with this, the induction of Fabp4 mRNA in NIH-PPAR $\gamma$ cells was also more robust than that of Nprc after rosiglitazone treatment (Fig. 2D). Second, while Nprc expression was increased in the brown fat of HFD-fed mice, Fabp4 mRNA was actually lower even though its $-5.4 \mathrm{~kb}$ PPAR $\gamma$ enhancer activity was unaffected by the HFD compared to chow. This again may be due to the fact that PPAR $\gamma$ could bind to both Fabp4 distal enhancer and proximal promoter $(21,35)$, so that the activity of the enhancer alone did not fully represent its overall mRNA expression levels. Moreover, it has been reported that FABP4 can attenuate PPAR $\gamma$ by triggering PPAR $\gamma$ ubiquitination and subsequent proteasomal degradation (36). Finally, while it appears that PPAR $\gamma$ is the major regulator of Fabp4 gene expression (20,21), Nprc gene expression is likely regulated by other factors in addition to PPAR $\gamma$ (29-32).

Our analysis of the long-range distal PPAR $\gamma$ enhancers only covered a $71 \mathrm{~kb}$ region upstream of Nprc promoter. However, enhancer elements may also reside in the downstream or intronic regions. As discussed by others (37), these enhancer elements do not necessarily act on the respective closest promoter, but can bypass neighboring genes to regulate genes located more distantly along a chromosome. As such we cannot exclude the possibility that additional long-range distal elements may exist and function synergistically with the $-49 \mathrm{~kb}$ and $-54 \mathrm{~kb}$ enhancers that we identified in this study to control Nprc gene expression. Unbiased approaches, such as the circular chromosome conformation capture assay (38), will be required to identify these additional elements in future study.

\section{The physiological significance of PPAR $\gamma$-dependent regulation of Nprc expression}

Our study presents the first evidence to directly link Nprc gene expression to PPAR $\gamma$, the master regulator of adipocyte formation. This connection may, at least partially, explain the well appreciated fact that Nprc is abundantly expressed in adipocytes and in the adipose tissue $(6,15)$. It will be interesting to determine whether Nprc expression could also be regulated by PPAR $\gamma$ in the other related tissues, such as the macrophage and the cardiovascular system (39-41). In addition, we observed that Nprc expression was significantly higher in the male epidydimal white adipose tissue than in the female parametrial white adipose tissue, and this difference was mirrored by the PPAR $\gamma$ enhancer activity (data not shown). This apparent sexually dimorphic and depot-specific pattern of PPAR $\gamma$ activity needs further investigation. 
It is well known that activation of PPAR $\gamma$ improves insulin sensitivity through a combination of metabolic actions, including partitioning of lipid stores and the regulation of metabolic and inflammatory mediators such as adiponectin (42). Our study showed that HFD-dependent Nprc expression could be potentially mediated by the action of PPAR $\gamma$. An increase in NPRC expression in the adipose tissue results in reduced NPRA to NPRC ratio and thus suppresses adipose tissue natriuretic peptide signaling in obesity. As we previously showed, deletion of Nprc in the adipose tissue enhances NP signaling, stimulates energy expenditure, improves glucose homeostasis and protects against diet-induced obesity $(6,16)$. Given the substantial role of NPRC for the control of natriuretic peptide signaling, our study provides a new connection to PPAR $\gamma$ for modulating natriuretic peptide signaling and energy metabolism during obesity.

A recently published promoter Capture Hi-C data set from human adipose tissue suggested several promoter interacting elements for the human NPRC gene (43). Motif and integration analysis with human adipocyte PPAR $\gamma$ ChIP-seq data suggested the existence of PPAR $\gamma$ binding sites within these NPRC promoterinteracting sequences. This indicates that NPRC expression may also be regulated by long-range distal PPAR $\gamma$ enhancers in human adipocytes. Furthermore, several studies including ours have demonstrated that adipose NPRC levels are increased with obesity in both mouse and human (9,13-18), thus the obesityassociated increase in NPRC expression is conserved between mouse and human. However, PPAR $\gamma-$ mediated NPRC gene expression in human adipocytes and adipose tissue will need to be further investigated. In conclusion, our current study demonstrates that PPAR $\gamma$ regulates Nprc expression in adipocytes through its long-range distal enhancers, and the diet-dependent increase in Nprc expression in obesity is potentially mediated by the actions of PPAR $\gamma$. Although we expect that PPAR $\gamma$ is not the sole regulator of Nprc expression in adipocytes, our study provides the first insight into the transcriptional regulation of the Nprc gene by establishing a new connection for how PPAR $\gamma$ may control natriuretic peptide signaling in the adipocyte.

\section{Material and Methods}

\section{Cell lines}

Mouse 3T3-L1 preadipocytes were maintained in DMEM supplemented with $10 \%$ calf serum, $100 \mathrm{U} / \mathrm{ml}$ penicillin and $100 \mu \mathrm{g} / \mathrm{ml}$ streptomycin. $\mathrm{C} \mathrm{H}_{10} \mathrm{~T}_{1 / 2}$ preadipocytes were maintained in DMEM supplemented with $10 \%$ fetal bovine serum (FBS), $100 \mathrm{U} / \mathrm{ml}$ penicillin and $100 \mu \mathrm{g} / \mathrm{ml}$ streptomycin. When reaching confluence, adipocyte differentiation was induced by a cocktail of $5 \mu \mathrm{g} / \mathrm{ml}$ insulin, $1 \mu \mathrm{M}$ dexamethasone, $0.5 \mathrm{mM}$ isobutyl methylxanthine, $1 \mu \mathrm{M}$ rosiglitazone in DMEM with $10 \%$ FBS for 3 days, then maintained 
in DMEM with 10\% FBS until day 6-7 when ready for experiment. NIH-3T3 fibroblasts and a clonal line that stably expresses PPAR $\gamma(\mathrm{NIH}-\mathrm{PPAR} \gamma)$ were cultured as described $(23,24)$.

\section{SiRNA}

Knockdown of Ppary in differentiating 3T3-L1 adipocytes was performed with Ppary esiRNA (Sigma, EMU041151) as previously described (44). Briefly, at day 4 of differentiation per the above protocol, 3T3L1 adipocytes were trypsinized and resuspended in growth medium at a density of $2.4 \times 10^{6}$ cells $/ \mathrm{ml}$. A mixture of 40 pmol siRNA and $24 \mu 1$ RNAiMAX (Invitrogen) were prepared with OptiMEM medium in a final volume of $160 \mu \mathrm{l}$, incubated at room temperature for $5 \mathrm{~min}$ and then added to a collagen (Gibco, A10644-01)-coated well of a 12-well plate. 3T3-L1 adipocytes were plated onto the 12-well plates at a density of $4.64 \times 10^{5}$ cells with a final media volume of $0.8 \mathrm{ml}$ per well for $6 \mathrm{hrs}$. After this step, cells were replaced with fresh media and cultured for another two days before harvesting for RNA and Western blot analysis.

\section{Chromosomal conformation capture assay (3C)}

$3 \mathrm{C}$ was performed according to previous reports (45-47). Briefly, $1 \times 10^{7} \mathrm{C}_{3} \mathrm{H}_{10 \mathrm{~T}_{1 / 2}}$ adipocytes at day 6 of differentiation were trypsinized, cross-linked by $2 \%$ formaldehyde at room temperature (RT) for $10 \mathrm{~min}$ and quenched with $0.125 \mathrm{M}$ glycine at RT for $5 \mathrm{~min}$. Adipocytes were washed and lysed in a buffer (10mM Tris- $\mathrm{HCl}$ pH8.0, 10mM NaCl, 0.2\% NP-40, 1x cOmplete Protease Inhibitor Cocktail from Roche) on ice for $30 \mathrm{~min}$ with shaking. The nuclei were harvested and resuspended in $0.5 \mathrm{ml}$ cold 1.2x NEB Buffer 2 (New England Biolabs), incubated with $0.3 \%$ SDS for $1 \mathrm{hr}$ at $37^{\circ} \mathrm{C}$, and with $1.8 \%$ Triton X-100 for $1 \mathrm{hr}$ at $37^{\circ} \mathrm{C}$. The isolated chromatin was digested with $1500 \mathrm{U}$ of HindIII (NEB) overnight with shaking at $37^{\circ} \mathrm{C}$ and then incubated with $1.6 \% \mathrm{SDS}$ at $65^{\circ} \mathrm{C}$ for $20 \mathrm{~min}$. The samples were transferred to $7 \mathrm{ml}$ of $1.15 \mathrm{x} \mathrm{T} 4$ ligation buffer (NEB), incubated with $1 \%$ Triton $\mathrm{X}-100$ for 1 hour at $37^{\circ} \mathrm{C}$, then ligated with $800 \mathrm{U}$ T4 ligase (NEB) for 4 hours at $16^{\circ} \mathrm{C}$ followed by 30min at RT. The chromatin was then digested with $300 \mu \mathrm{g}$ Proteinase $\mathrm{K}$ overnight at $65^{\circ} \mathrm{C}$ and by $30 \mu \mathrm{g}$ RNaseA for 1 hour at $37^{\circ} \mathrm{C}$. The DNA was purified with phenol/chloroform extraction, precipitated with ethanol, dissolved in $150 \mu$ l of Tris buffer (10mM, pH7.5), and quantified with NanoDrop (Thermo Scientific). Two hundred ng DNA sample was used for PCR with an anchor primer in the Nprc promoter fragment and a bait primer in each of the distal enhancer fragments to determine the Nprc promoter and enhancer interaction (Fig. 3B). The 3C PCR primers are listed in the Supplementary Table. For positive controls, BAC DNA that cover Nprc (RP23-305L10) and Ercc3 (RP23148C24) genes were digested with HindIII and randomly religated to generate all possible ligation products as described previously (47).

\section{Luciferase reporter assays}


A $-2.2 \mathrm{~kb}$ fragment of the mouse Nprc gene promoter (-2233bp to $+1 \mathrm{bp}, m N p r c-2.2 \mathrm{k}$ ) was amplified by PCR with overhangs in the forward (5'-ATGACTCGAG-3') and reverse (5'-ATTAAAGCTT-3') primers, digested with XhoI and HindIII and cloned into pGL-4.14 vector. The positive control plasmid with a PPAR $\gamma$ enhancer from Angptl4 gene in pUC18 HSV TK-Luc vector (Angptl4+2.3k) was constructed as described $(27,48)$. The individual enhancer fragments were amplified by PCR with overhangs in the forward (5'-ATTGTTGGATCC-3') and reverse (5'-ATTGTTGTCGAC-3') primers, digested with SalI and BamHI and cloned into pUC18 HSV TK-Luc vector. The reporter plasmid cloning primers are listed in the Supplementary Table. For luciferase assay, NIH-3T3 and NIH-PPAR $\gamma$ fibroblasts were plated in 12well culture plates at a density of $1.2 \times 10^{5}$ cells/well and reached $80 \%$ confluence the following day when ready for transfection. Cells were transfected with $1.25 \mu \mathrm{g} /$ well of the reporter plasmids by polyethyleneimine reagent (Polysciences, \#23966) and differentiated in DMEM/10\% FBS with $1 \mu \mathrm{M}$ rosiglitazone for two days. Luciferase activity was assayed according to the manufacturer's instruction (Promega, E1501) and normalized to total protein concentration (BCA, Pierce).

\section{High-fat diet (HFD) experiment}

8-week-old wild-type male mice were fed with a high-fat diet (60\% of calories from fat, Research Diet, D12492) for 12 weeks. All animal studies were approved by the Institutional Animal Care and Use Committee of Vanderbilt University Medical Center and in accordance with the NIH Guide for the Care and Use of Laboratory Animals.

\section{QPCR}

RNA was extracted with TRIzol (Invitrogen), purified with RNeasy Mini columns (Qiagen) or Quick-RNA columns (Zymo Research) and reversed transcribed with High-Capacity cDNA Reverse Transcription Kits (Applied Biosystems). For enhancer RNA evaluation, cDNA was prepared with iScript gDNA Clear cDNA Synthesis Kit (Bio-Rad) to avoid genomic DNA contamination. QPCR was performed with PowerUp SYBR Green Master Mix (Life Technologies) according to manufacturer's instruction. QPCR results were analyzed with the $\Delta \Delta \mathrm{Ct}$ method, normalized to the internal control gene 36B4 and presented as fold change relative to the control group. QPCR primers are listed in Supplementary Table.

\section{Western blot}

Protein was extracted from cells as previously described (49). For Western blotting analysis, 30-40 $\mu \mathrm{g}$ of protein was resolved by $10 \%$ SDS-polyacrylamide gel electrophoresis, transferred to nitrocellulose membranes (Bio-Rad), incubated overnight at $4^{\circ} \mathrm{C}$ with specific primary antibodies in blocking buffer (TrisBuffered Saline, $0.1 \%$ Tween-20, 5\% milk or BSA) and detected with alkaline phosphatase (AP)conjugated secondary antibody. The antibodies used were anti-NPRC (1:2000, Novus Biologicals, \#NBP1- 
31365), anti-PPAR $\gamma$ (1:2000, Cell Signaling Technology, \#2435), anti-FABP4 (1:1000, Cell Signaling Technology, \#2120), anti-ADIPOQ (1:1000, Cell Signaling Technology, \#2789), anti-GAPDH ( 1:2000, Protein Tech, \#10494-1-AP) and anti-rabbit IgG-AP (1:20000, Sigma, \#A3687)

\section{ChIP-seq data analysis}

A previously published PPAR $\gamma$ ChIP-seq dataset in 3T3-L1 adipocyte and adipose tissues (Gene Expression Omnibus, GSE92606) (25) was downloaded and analyzed with Integrative Genomics Viewer (Broad Institute) (50).

\section{Statistical analysis}

GraphPad Prism 7 was used for statistical analysis. All data were presented as means \pm SEM. Unpaired two-tailed Student's t-tests were used to determine the differences between groups. Statistical significance was defined as p-value $<0.05$.

\section{Acknowledgements}

The authors thank Ms. Wei Zhang for animal husbandry assistance, Dr. Ryan P. Ceddia for advice and comments on the manuscript, and Dr. Stephen Farmer for the NIH-3T3 and NIH-PPAR $\gamma$ cells.

\section{Author Contributions}

S.C. supervised the project. F.S. designed and performed experiments and analyzed the data. Z.S. and L.N. assisted with ChIP-seq and enhancer RNA analysis. F.S. and S.C. wrote the manuscript.

\section{Funding and Additional Information}

This work was supported by NIH R01 DK103056 (S.C.), R56 DK126890 (S.C.) and R01 DK115924 (L.N.). F.S. is supported by the America Diabetes Association Postdoctoral Fellowship Award (1-18-PDF110). The content is solely the responsibility of the authors and does not necessarily represent the official views of the National Institutes of Health. 


\section{Supplementary Table: Primer sequences}

\begin{tabular}{|c|c|c|}
\hline QPCR & Fwd (5'-3') & $\operatorname{Rev}\left(5^{\prime}-3^{\prime}\right)$ \\
\hline 36B4 & GATGCCCAGGGAAGACAG & ACAATGAAGCATTTTGGATAATCA \\
\hline Nprc & AGCTGGCTACAGCAAGAAGG & CGGCGATACCTTCAAATGTC \\
\hline Pparg2 & TGCTGTTATGGGTGAAACTCTG & CTGTGTCAACCATGGTAATTTCTT \\
\hline Pparg & GAAAGACAACGGACAAATCACC & GGGGGTGATATGTTTGAACTTG \\
\hline Fabp4 & GGATGGAAAGTCGACCACAA & TGGAAGTCACGCCTTTCATA \\
\hline Npra & TGGAGACACAGTCAACACAGC & CGAAGACAAGTGGATCCTGAG \\
\hline$\underline{\text { 3C PCR }}$ & Enhancer Bait (5'-3') & Promoter Anchor (5'-3') \\
\hline Nprc-9k & GTCTGTATTTCTGAGGAGTGCTGTA & AGCCTTCCTTTTAGATTCCTTCCAT \\
\hline Nprc-44/49k & GTTGTTTCCCTGAATTCCTAAACCC & AGCCTTCCTTTTAGATTCCTTCCAT \\
\hline Nprc-51k & CTTCTCATGGCCTTTGTGTGTTAAT & AGCCTTCCTTTTAGATTCCTTCCAT \\
\hline Nprc-54k & TACTGTTCAAGCCATCACAGAACTA & AGCCTTCCTTTTAGATTCCTTCCAT \\
\hline Nprc-58k & TCCTGTAGCTGTCCAGTCCA & AGCCTTCCTTTTAGATTCCTTCCAT \\
\hline Nprc-62k & TGCTAACTGGATGCCACTGG & AGCCTTCCTTTTAGATTCCTTCCAT \\
\hline Nprc-71k & CTCATGAAGGAGGGACAGGAGGAGCTG & AGCCTTCCTTTTAGATTCCTTCCAT \\
\hline Ercc3 & CACATGCCCTCCCTGAAAATAAG & CTAAAGAAGAGCAGGAGGTTTCAGA \\
\hline$\beta$-actin & CTGTCGAGTCGCGTCCACCC & CCGCACCGGCTCATCAAATG \\
\hline$\underline{\text { Reporters }}$ & Fwd (5'-3') & $\operatorname{Rev}\left(5^{\prime}-3^{\prime}\right)$ \\
\hline Nprc-2.2k & ATGACTCGAGCCGGCACCCGGTATGAAGTT & ATTAAAGCTTCTCGCCCTCTGCCCCCCAC \\
\hline Nprc-9k & ATGCGTCGACTGGAGGAGGCTTTGAGATCC & ATGCGGATCCGTCCCACACCAAGACGTAGA \\
\hline Nprc-44k & ATGCAAGCTTAGCTGCAGGATCTCTACGAC & ATGCGTCGACGGTAGAGGCAGGTGGATCTC \\
\hline Nprc-49k & ATGCGTCGACAGCAAAGCATACTTGCTGGGAAG & ATGCGGATCCAAGAGAGCTGGGACCTTCAGGAC \\
\hline Nprc-51k & ATTGTTGTCGACGCGCCAGGAAGTAAGTTGAG & ATTGTTGGATCCGCTGAACATGCTGGTTTCCA \\
\hline Nprc-54k & ATGCGTCGACCTCAATGAACTTCTATTTAGTTTGTATCAC & ATGCGGATCCTGGTCATTATTTTCCACTTGAGCAGC \\
\hline Nprc-58k & ATGCGTCGACTGTCTCCCTCCCTCCATGTA & ATGCGGATCCCTTGGTTTTCACTTTCAGTTCAGT \\
\hline Nprc-62k & ATGCGTCGACAGGAATGGACAGAAAG & ATGCGGATCCATTTTGAAGTCGGGCTGGTG \\
\hline Nprc-49k-P1 & ATTGTTGTCGACAGTTTGCAAGAAGAAAGACAGCT & ATTGTTGGATCCACTCCTGAAAGTTGTCCTCTGAT \\
\hline Nprc-49k-P2 & ATTGTTGTCGACATACAAACAGGAACCTCAACTCC & ATTGTTGGATCCCCCAAGTCAGCAGCTAATCATAG \\
\hline Nprc-49k-P3 & ATTGTTGTCGACGTTGAGGGAAGGACATTGATCTG & ATTGTTGGATCCACACCACAAGAGATAGCCAGATA \\
\hline Nprc-54k-P4 & ATTGTTGTCGACGGATCAGGTGTTCAGGGCTA & ATTGTTGGATCCATGCATTGGTGGTGTTTTACAA \\
\hline
\end{tabular}




\section{References}

1. de Bold, A. J., Borenstein, H. B., Veress, A. T., and Sonnenberg, H. (1981) A rapid and potent natriuretic response to intravenous injection of atrial myocardial extract in rats. Life Sci 28, 89-94

2. Ogawa, T., Linz, W., Stevenson, M., Bruneau, B. G., Kuroski de Bold, M. L., Chen, J. H., Eid, H., Scholkens, B. A., and de Bold, A. J. (1996) Evidence for load-dependent and loadindependent determinants of cardiac natriuretic peptide production. Circulation 93, 2059-2067

3. $\quad$ Schlueter, N., de Sterke, A., Willmes, D. M., Spranger, J., Jordan, J., and Birkenfeld, A. L. (2014) Metabolic actions of natriuretic peptides and therapeutic potential in the metabolic syndrome. Pharmacol Ther 144, 12-27

4. Sengenes, C., Berlan, M., De Glisezinski, I., Lafontan, M., and Galitzky, J. (2000) Natriuretic peptides: a new lipolytic pathway in human adipocytes. FASEB J 14, 1345-1351

5. Sengenes, C., Zakaroff-Girard, A., Moulin, A., Berlan, M., Bouloumie, A., Lafontan, M., and Galitzky, J. (2002) Natriuretic peptide-dependent lipolysis in fat cells is a primate specificity. Am J Physiol Regul Integr Comp Physiol 283, R257-265

6. Bordicchia, M., Liu, D., Amri, E. Z., Ailhaud, G., Dessi-Fulgheri, P., Zhang, C., Takahashi, N., Sarzani, R., and Collins, S. (2012) Cardiac natriuretic peptides act via p38 MAPK to induce the brown fat thermogenic program in mouse and human adipocytes. J. Clin. Invest. 122, 1022-1036

7. Potter, L. R. (2011) Natriuretic peptide metabolism, clearance and degradation. FEBS J 278, 1808-1817

8. Dessi-Fulgheri, P., Sarzani, R., and Rappelli, A. (1998) The natriuretic peptide system in obesityrelated hypertension: new pathophysiological aspects. J Nephrol 11, 296-299

9. Wang, T. J., Larson, M. G., Levy, D., Benjamin, E. J., Leip, E. P., Wilson, P. W., and Vasan, R. S. (2004) Impact of obesity on plasma natriuretic peptide levels. Circulation 109, 594-600

10. Fox, E. R., Musani, S. K., Bidulescu, A., Nagarajarao, H. S., Samdarshi, T. E., Gebreab, S. Y., Sung, J. H., Steffes, M. W., Wang, T. J., Taylor, H. A., and Vasan, R. S. (2011) Relation of obesity to circulating B-type natriuretic peptide concentrations in blacks: the Jackson Heart Study. Circulation 124, 1021-1027

11. Clerico, A., Giannoni, A., Vittorini, S., and Emdin, M. (2012) The paradox of low BNP levels in obesity. Heart Failure Reviews 17

12. Matsukawa, N., Grzesik, W. J., Takahashi, N., Pandey, K. N., Pang, S., Yamauchi, M., and Smithies, O. (1999) The natriuretic peptide clearance receptor locally modulates the physiological effects of the natriuretic peptide system. Proc Natl Acad Sci U S A 96, 7403-7408

13. Dessi-Fulgheri, P., Sarzani, R., Tamburrini, P., Moraca, A., Espinosa, E., Cola, G., Giantomassi, L., and Rappelli, A. (1997) Plasma atrial natriuretic peptide and natriuretic peptide receptor gene expression in adipose tissue of normotensive and hypertensive obese patients. J Hypertens $\mathbf{1 5}$, 1695-1699

14. Sarzani, R., Strazzullo, P., Salvi, F., Iacone, R., Pietrucci, F., Siani, A., Barba, G., Gerardi, M. C., Dessi-Fulgheri, P., and Rappelli, A. (2004) Natriuretic peptide clearance receptor alleles and susceptibility to abdominal adiposity. Obes Res 12, 351-356

15. Kovacova, Z., Tharp, W. G., Liu, D., Wei, W., Xie, H., Collins, S., and Pratley, R. E. (2016) Adipose tissue natriuretic peptide receptor expression is related to insulin sensitivity in obesity and diabetes. Obesity (Silver Spring) 24, 820-828

16. Wu, W., Shi, F., Liu, D., Ceddia, R. P., Gaffin, R., Wei, W., Fang, H., Lewandowski, E. D., and Collins, S. (2017) Enhancing natriuretic peptide signaling in adipose tissue, but not in muscle, protects against diet-induced obesity and insulin resistance. Sci Signaling 10, eaam6870

17. Coue, M., Badin, P. M., Vila, I. K., Laurens, C., Louche, K., Marques, M. A., Bourlier, V., Mouisel, E., Tavernier, G., Rustan, A. C., Galgani, J. E., Joanisse, D. R., Smith, S. R., Langin, D., and Moro, C. (2015) Defective natriuretic peptide receptor signaling in skeletal muscle links obesity to Type 2 diabetes. Diabetes 64, 4033-4045 
18. de Andrade, E. N., Goncalves, G. K., de Oliveira, T. H., Santos, C. S., Souza, C. L., Firmes, L. B., de Magalhaes, A. C., Soares Tde, J., Reis, A. M., and Belo Nde, O. (2011) Natriuretic peptide system: a link between fat mass and cardiac hypertrophy and hypertension in fat-fed female rats. Regul Pept 167, 149-155

19. Tontonoz, P., Hu, E., and Spiegelman, B. M. (1994) Stimulation of adipogenesis in fibroblasts by PPARg2, a lipid-activated transcription factor. Cell 79, 1147-1156

20. Tontonoz, P., Hu, E., Graves, R. A., Budavari, A. I., and Spiegelman, B. M. (1994) mPPAR gamma 2: tissue-specific regulator of an adipocyte enhancer. Genes Dev. 8, 1224-1234

21. Tontonoz, P., Graves, R. A., Budavari, A. I., Erdjument-Bromage, H., Lui, M., Hu, E., Tempst, P., and Spiegelman, B. M. (1994) Adipocyte-specific transcription factor ARF6 is a heterodimeric complex of two nuclear hormone receptors, PPAR gamma and RXR alpha. Nucleic Acids Research 22, 5628-5634

22. Maeda, N., Takahashi, M., Funahashi, T., Kihara, S., Nishizawa, H., Kishida, K., Nagaretani, H., Matsuda, M., Komuro, R., Ouchi, N., Kuriyama, H., Hotta, K., Nakamura, T., Shimomura, I., and Matsuzawa, Y. (2001) PPARgamma ligands increase expression and plasma concentrations of adiponectin, an adipose-derived protein. Diabetes 50, 2094-2099

23. Wu, Z., Xie, Y., Morrison, R. F., Bucher, N. L., and Farmer, S. R. (1998) PPARgamma induces the insulin-dependent glucose transporter GLUT4 in the absence of C/EBPalpha during the conversion of $3 \mathrm{~T} 3$ fibroblasts into adipocytes. J. Clin. Invest. 101, 22-32

24. Dixon, T. M., Daniel, K. W., Farmer, S. R., and Collins, S. (2001) CAATT/Enhancer binding protein-a is required for transcription of the $\mathrm{b}_{3}$ AR gene during adipogenesis. J. Biol. Chem. 276, $722-728$

25. Soccio, R. E., Li, Z., Chen, E. R., Foong, Y. H., Benson, K. K., Dispirito, J. R., Mullican, S. E., Emmett, M. J., Briggs, E. R., Peed, L. C., Dzeng, R. K., Medina, C. J., Jolivert, J. F., Kissig, M., Rajapurkar, S. R., Damle, M., Lim, H. W., Won, K. J., Seale, P., Steger, D. J., and Lazar, M. A. (2017) Targeting PPAR $\gamma$ in the epigenome rescues genetic metabolic defects in mice. J. Clin. Invest. 127, 1451-1462

26. Schoenfelder, S., and Fraser, P. (2019) Long-range enhancer-promoter contacts in gene expression control. Nat Rev Genet 20, 437-455

27. Daniel, B., Nagy, G., Hah, N., Horvath, A., Czimmerer, Z., Poliska, S., Gyuris, T., Keirsse, J., Gysemans, C., Van Ginderachter, J. A., Balint, B. L., Evans, R. M., Barta, E., and Nagy, L. (2014) The active enhancer network operated by liganded RXR supports angiogenic activity in macrophages. Genes Dev 28, 1562-1577

28. Li, W., Notani, D., and Rosenfeld, M. G. (2016) Enhancers as non-coding RNA transcription units: recent insights and future perspectives. Nat Rev Genet 17, 207-223

29. Bordicchia, M., Ceresiani, M., Pavani, M., Minardi, D., Polito, M., Wabitsch, M., Cannone, V., Burnett, J. C., Jr., Dessi-Fulgheri, P., and Sarzani, R. (2016) Insulin/glucose induces natriuretic peptide clearance receptor in human adipocytes: a metabolic link with the cardiac natriuretic pathway. Am J Physiol Regul Integr Comp Physiol 311, R104-114

30. Harvell, D. M., Richer, J. K., Allred, D. C., Sartorius, C. A., and Horwitz, K. B. (2006) Estradiol regulates different genes in human breast tumor xenografts compared with the identical cells in culture. Endocrinology 147, 700-713

31. Kishimoto, I., Yoshimasa, T., Suga, S., Ogawa, Y., Komatsu, Y., Nakagawa, O., Itoh, H., and Nakao, K. (1994) Natriuretic peptide clearance receptor is transcriptionally down-regulated by beta 2-adrenergic stimulation in vascular smooth muscle cells. J. Biol. Chem. 269, 28300-28308

32. Pivovarova, O., Gogebakan, O., Kloting, N., Sparwasser, A., Weickert, M. O., Haddad, I., Nikiforova, V. J., Bergmann, A., Kruse, M., Seltmann, A. C., Bluher, M., Pfeiffer, A. F., and Rudovich, N. (2012) Insulin up-regulates natriuretic peptide clearance receptor expression in the subcutaneous fat depot in obese subjects: a missing link between CVD risk and obesity? J Clin Endocrinol Metab 97, E731-739 
33. Lehrke, M., and Lazar, M. A. (2005) The many faces of PPARgamma. Cell 123, 993-999

34. Xu, H. E., and Li, Y. (2008) Ligand-dependent and -independent regulation of PPAR gamma and orphan nuclear receptors. Sci Signal 1, pe52

35. Shin, J., Li, B., Davis, M. E., Suh, Y., and Lee, K. (2009) Comparative analysis of fatty acidbinding protein 4 promoters: conservation of peroxisome proliferator-activated receptor binding sites. J. Animal Sci. 87, 3923-3934

36. Garin-Shkolnik, T., Rudich, A., Hotamisligil, G. S., and Rubinstein, M. (2014) FABP4 attenuates PPAR $\gamma$ and adipogenesis and is inversely correlated with PPAR $\gamma$ in adipose tissues. Diabetes $6 \mathbf{6 3}$ 900-911

37. Pennacchio, L. A., Bickmore, W., Dean, A., Nobrega, M. A., and Bejerano, G. (2013) Enhancers: five essential questions. Nat Rev Genetics 14, 288-295

38. Gö ndö r, A., Rougier,C., and Ohlsson, R. (2008) High-resolution circular chromosome conformation capture assay. Nature Protoc 3, 303-313

39. Duan, S. Z., Ivashchenko, C. Y., Russell, M. W., Milstone, D. S., and Mortensen, R. M. (2005) Cardiomyocyte-specific knockout and agonist of peroxisome proliferator-activated receptor- $\gamma$ both induce cardiac yypertrophy in mice. Circulation Research 97, 372-379

40. Babaev, V. R., Yancey, P. G., Ryzhov, S. V., Kon, V., Breyer, M. D., Magnuson, M. A., Fazio, S., and Linton, M. F. (2005) Conditional knockout of macrophage PPAR $\gamma$ increases atherosclerosis in C57BL/6 and low-density lipoprotein receptor-deficient mice. Arterio. Thromb. Vasc. Biol. 25, 1647-1653

41. Hsueh, W. A., and Bruemmer, D. (2004) Peroxisome proliferator-activated receptor $\gamma$ : implications for cardiovascular disease. Hypertension 43, 297-305

42. Tontonoz, P., and Spiegelman, B. M. (2008) Fat and beyond: the diverse biology of PPARgamma. Annu Rev Biochem 77, 289-312

43. Pan, D. Z., Garske, K. M., Alvarez, M., Bhagat, Y. V., Boocock, J., Nikkola, E., Miao, Z., Raulerson, C. K., Cantor, R. M., Civelek, M., Glastonbury, C. A., Small, K. S., Boehnke, M., Lusis, A. J., Sinsheimer, J. S., Mohlke, K. L., Laakso, M., Pajukanta, P., and Ko, A. (2018) Integration of human adipocyte chromosomal interactions with adipose gene expression prioritizes obesity-related genes from GWAS. Nature Comm. 9, 1512

44. Kilroy, G., Burk, D. H., and Floyd, Z. E. (2009) High efficiency lipid-based siRNA transfection of adipocytes in suspension. PLoS One 4, e6940

45. Hagege, H., Klous, P., Braem, C., Splinter, E., Dekker, J., Cathala, G., de Laat, W., and Forne, T. (2007) Quantitative analysis of chromosome conformation capture assays (3C-qPCR). Nat Protoc 2, 1722-1733

46. Cope, N. F., and Fraser, P. (2009) Chromosome conformation capture. Cold Spring Harb Protoc 2009, pdb prot5137

47. Miele, A., Gheldof, N., Tabuchi, T. M., Dostie, J., and Dekker, J. (2006) Mapping chromatin interactions by chromosome conformation capture. Current Protocols in Molecular Biology Chapter 21, Unit 21.11

48. Szanto, A., Balint, B. L., Nagy, Z. S., Barta, E., Dezso, B., Pap, A., Szeles, L., Poliska, S., Oros, M., Evans, R. M., Barak, Y., Schwabe, J., and Nagy, L. (2010) STAT6 transcription factor is a facilitator of the nuclear receptor PPARgamma-regulated gene expression in macrophages and dendritic cells. Immunity 33, 699-712

49. Liu, D., Bordicchia, M., Zhang, C., Fang, H., Wei, W., Li, J. L., Guilherme, A., Guntur, K., Czech, M. P., and Collins, S. (2016) Activation of mTORC1 is essential for beta-adrenergic stimulation of adipose browning. J. Clin. Invest. 126, 1704-1716

50. Robinson, J. T., Thorvaldsdó ttir, H.,Winckler, W., Guttman, M., Lander, E. S., Getz, G., and Mesirov, J. P. (2011) Integrative genomics viewer. Nature Biotech 29, 24-26 

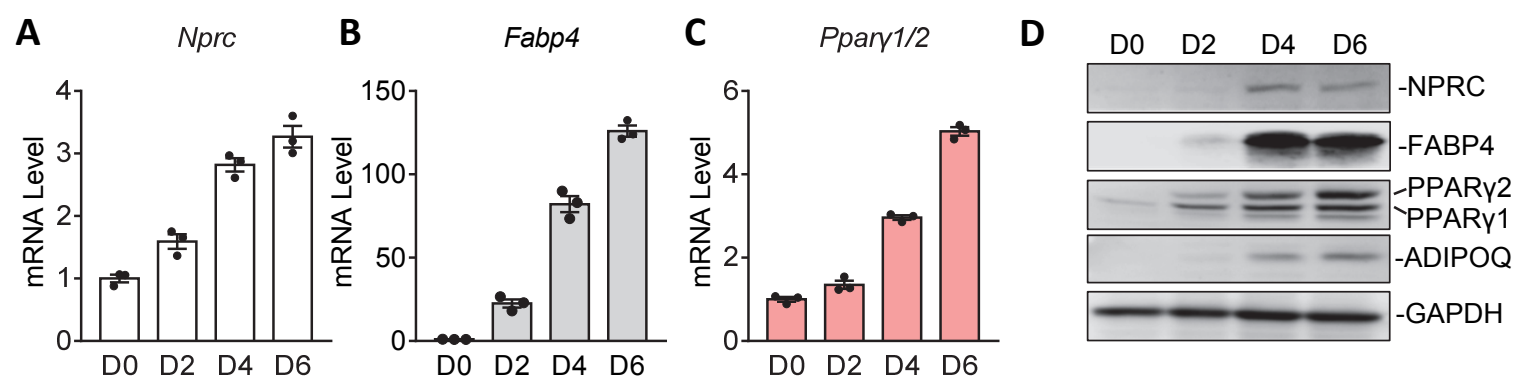

Figure 1. Nprc mRNA expression during 3T3-L1 adipocyte differentiation.

(A-C) mRNA levels of Nprc (A), Fabp4 (B) and Ppary1/2 (C) during 3T3-L1 adipocyte differentiation. Data were normalized with 36B4. (D) Protein levels of NPRC, FABP4, PPARY1/2 and ADIPOQ during 3T3-L1 adipocyte differentiation (Day 0, 2, 4 and 6). 


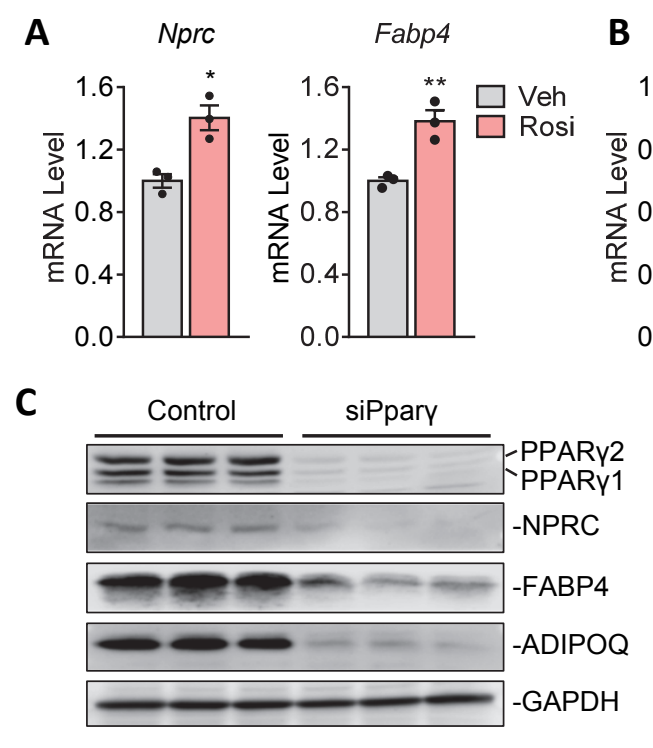

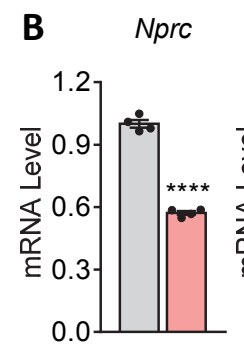
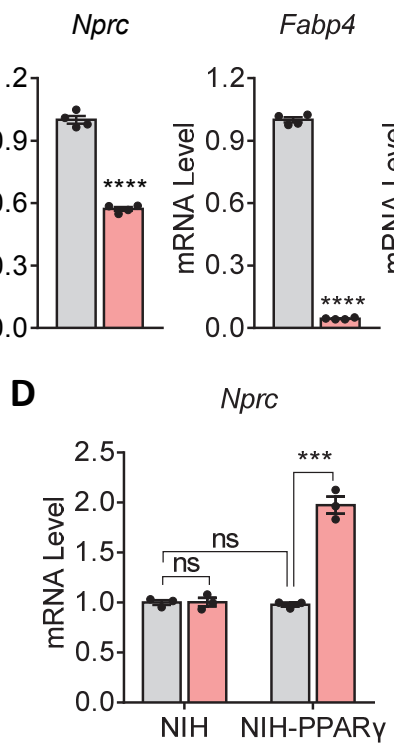
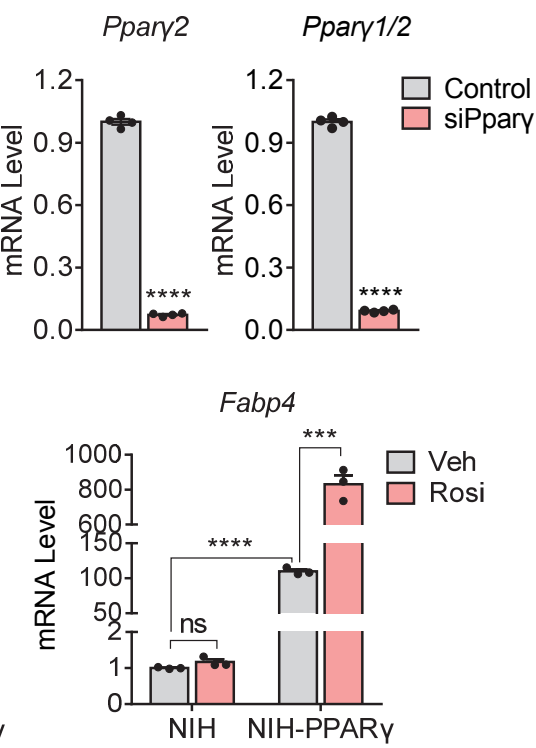

Figure 2. Modulation of Nprc expression by rosiglitazone and PPARy.

(A) mRNA levels of Nprc and Fabp4 in 3T3-L1 adipocytes after treatment with $1 \mu \mathrm{M}$ rosiglitazone (Rosi) or vehicle (Veh) for 6 hours. (B) mRNA levels of Nprc, Fabp4 and Ppary1/2 mRNA in 3T3-L1 adipocytes after siRNA knock down of Ppary (siPpary). (C) Protein levels of PPARy1/2, NPRC, FABP4 and ADIPOQ in 3T3-L1 adipocytes after siRNA knock down of Ppary (siPpary). (D) mRNA levels of Nprc and Fabp4 in NIH-3T3 cells $(\mathrm{NIH})$ and $\mathrm{NIH}-3 \mathrm{~T} 3$ cells stably expressing PPARY (NIH-PPARY) after treatment with $1 \mu \mathrm{M}$ rosiglitazone (Rosi) or vehicle (Veh) for 6 hours. QPCR data were normalized with 36B4. Student's t-test, ${ }^{*} p<0.05,{ }^{* *} p<0.01,{ }^{* * *} p<0.001,{ }^{* * * *} p<0.0001$, ns: not significant. 


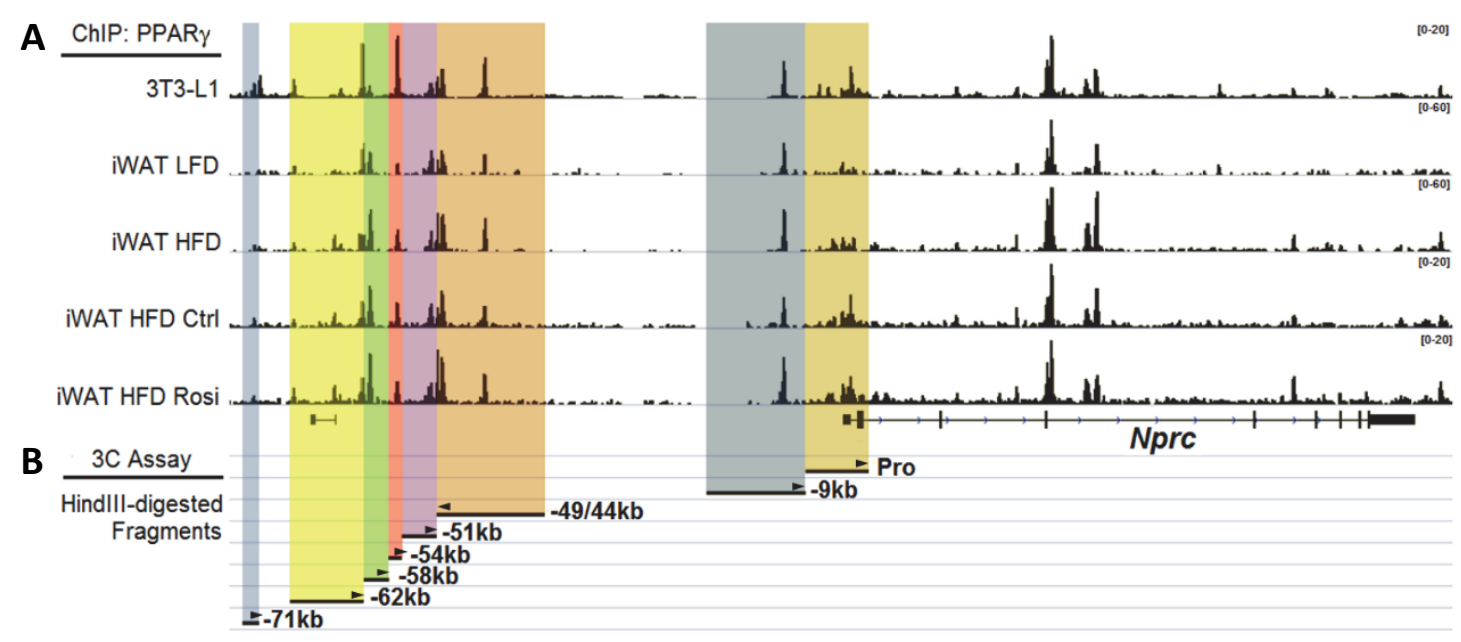

Figure 3. ChIP-seq identified PPARy enhancers in the upstream distal region of Nprc.

(A) PPARy binding sites in the upstream distal region of the Nprc promoter were identified by ChIP-seq in 3T3-L1 adipocytes and inguinal white adipose tssue (iWAT) from mice fed with low-fat diet (LFD), high-fat diet (HFD), HFD plus vehicle (Ctrl), and HFD plus rosiglitazone (Rosi) (Soccio et al., JCI, 2017). (B) Hindlll-digested restriction fragments containing Nprc promoter (Pro) and the PPARY binding sites (-9kb, $-44 / 49 \mathrm{~kb},-51 \mathrm{~kb},-54 \mathrm{~kb},-58 \mathrm{~kb}, 62 \mathrm{~kb}$ and $-71 \mathrm{~kb})$ are illustrated for the chromosomal conformation capture (3C) analysis in Figure 4. The arrowheads indicate the primers used for 3C PCR in each fragment. 

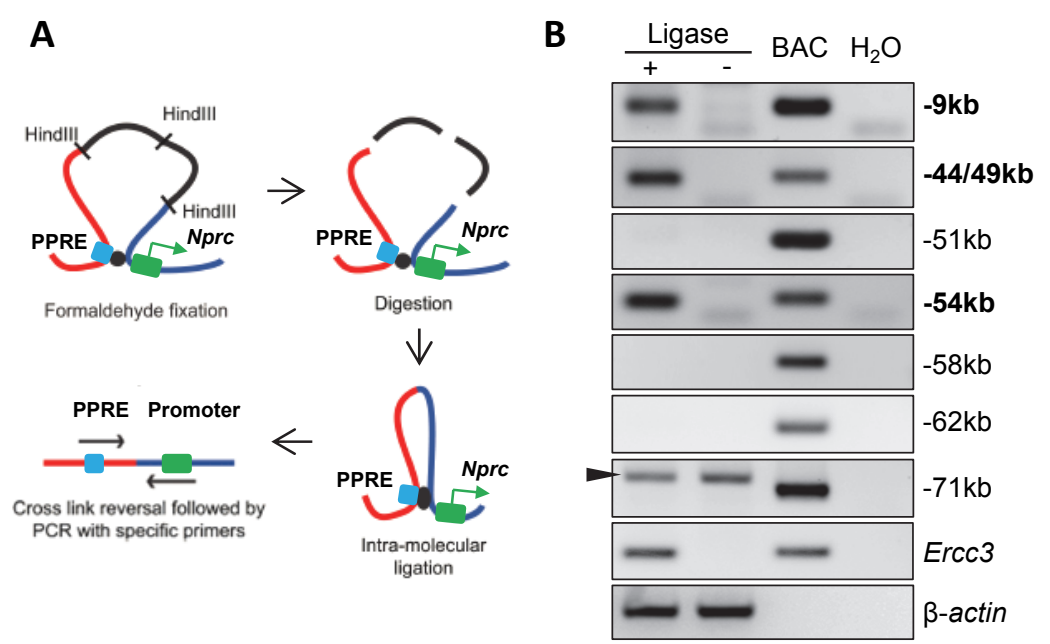

Figure 4. Interaction of PPARy-binding fragments with Nprc promoter.

(A) Procedure of chromosomal conformation analysis (3C) (modified from Cope and Fraser, 2009). PPRE: PPARy response elements. (B) $3 \mathrm{C}$ analysis with $\mathrm{C}_{3 \mathrm{H}} 10 \mathrm{~T}_{1 / 2}$ adipocytes to determine the interaction between Nprc promoter and PPARY-binding sites within each Hindlll-restriction fragment ($9 \mathrm{~kb},-44 / 49 \mathrm{~kb},-54 \mathrm{~kb},-58 \mathrm{~kb},-62 \mathrm{~kb}$ and $-71 \mathrm{~kb}$, also see Figure 3B for details). Ercc3 was positive control for constitutive enhancer interaction. $\beta$-actin was DNA input control. Ligase+, HindllI-digested chromatin with intramolecular religation. Ligase-, Hindlll-digested chromatin without intramolecular religation. BAC, bacterial artificial chromosome DNA covering Nprc (RP23-305L10) and Ercc3 (RP23148C24) genes was digested with HindIII and randomly religated as positive control. Arrowhead indicates non-specific amplification products. 


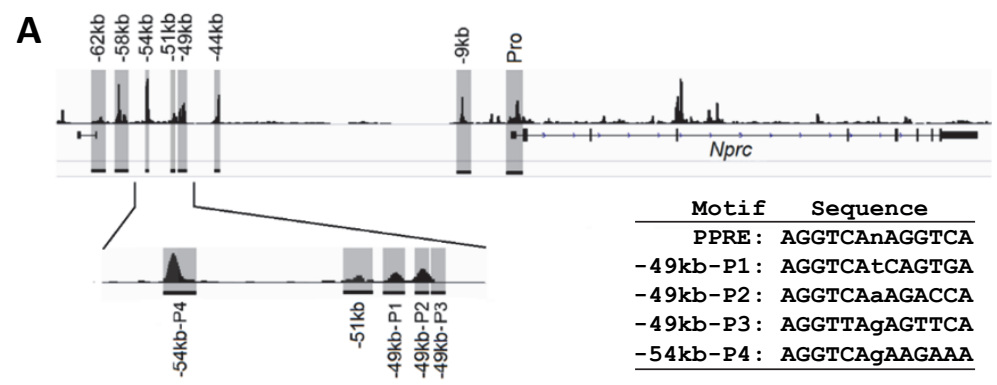

B

Nprc-2.2k Promoter

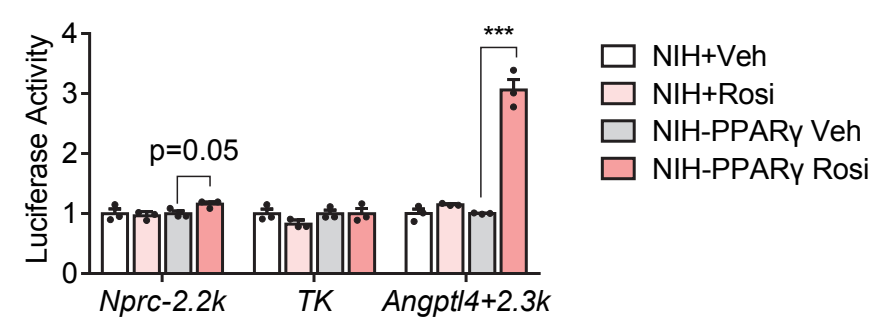

C

Nprc Distal Enhancers

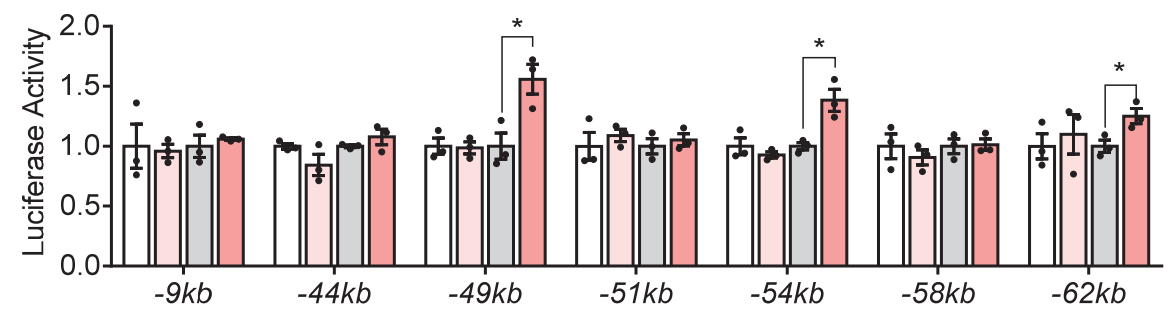

D

Nprc Distal PPREs

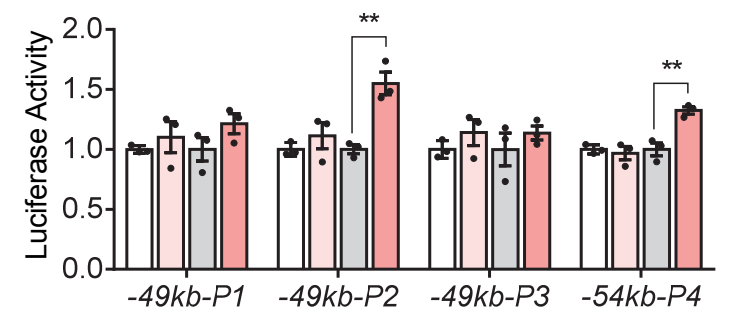

Figure 5. Rosiglitazone increases Nprc distal enhancer activity but not proximal promoter activity alone.

(A) Nprc promoter (Pro), distal PPARy enhancers (-9kb, -44kb, 49kb, -51kb, -54kb, -58kb, 62kb) and the four PPARy response elements (PPRE, P1, P2, P3 and P4) are shown in gray and were cloned for luciferase reporter analysis. The sequences of the consensus PPRE motif and the four Nprc PPREs are listed as insert. (B) Luciferase activity of Nprc promoter $(-2233 \mathrm{bp}$ to $+1 \mathrm{bp}, m N p r c-2.2 k)$. TK promoter alone $(T K)$ and TK promoter with a PPARy enhancer from the Angpt/4 gene (Angpt/4+2.3k) were used as the negative and positive controls, respectively. (C) Luciferase activity of the Nprc distal enhancer fragments (-9kb, -44kb, $-49 \mathrm{~kb},-51 \mathrm{~kb},-54 \mathrm{~kb},-58 \mathrm{~kb}$ and $-62 \mathrm{~kb})$. (D) Enhancer activity of the Nprc distal PPREs in the $-49 \mathrm{~kb}$ fragment (P1-P3) and $-54 \mathrm{~kb}$ fragment (P4). For reporter assays, NIH$3 \mathrm{~T} 3(\mathrm{NIH})$ and NIH-3T3 stably expressing PPARY (NIH-PPARY) cells were transfected with reporter plasmids and treated with vehicle (Veh) or $1 \mu \mathrm{M}$ rosiglitazone (Rosi) for 48 hours. Luciferase activity was normalized to protein concentrations. Data were representative of at least three independent experiments. Student t-test, ${ }^{*} p<0.05,{ }^{* *} p<0.01,{ }^{* * *} p<0.001$. 

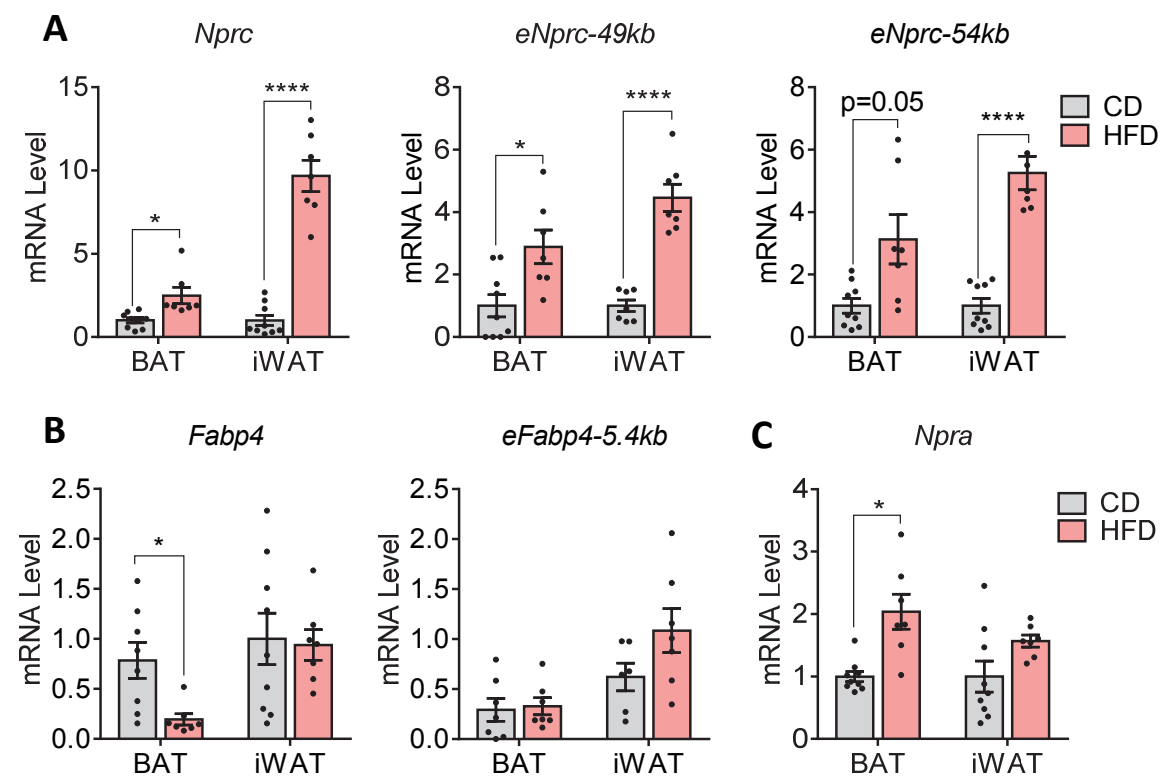

Figure 6. High-fat diet induces Nprc expression and PPARy enhancer activity in adipose tissue. (A) Levels of Nprc mRNA and the -49kb, -54kb PPARy enhancer RNAs (eNprc) in brown adipose tissue (BAT) and inguinal white adipose tissue (iWAT) of wild-type mice fed with control diet (CD) or high-fat diet (HFD). (B) Levels of Fabp4 and the -5.4kb PPARY enhancer RNA (eFabp4) in BAT and iWAT of mice fed with CD and HFD. (C) Levels of Npra mRNA in the BAT and iWAT of mice fed with CD and HFD. Student t-test, ${ }^{*} p<0.05,{ }^{* * * *} p<0.0001$, ns: not significant. 


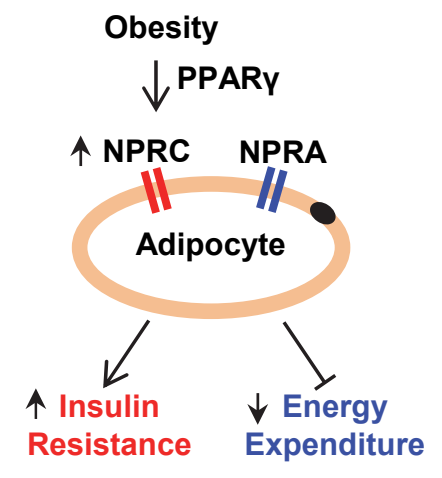

Figure 7. Obesity induces NPRC expression in adipocytes.

Obesity induces NPRC expression in adipocytes potentially though a PPARy-dependent mechanism. The increase in NPRC lowers the NPRA/NPRC ratio and reduces adipocyte natriuretic peptide signaling and results in decreased energy expenditure and impaired insulin sensitivity. 